\title{
Measuring Response of Extruded Scintillator to UV LED in Magnetic Field
}

\author{
D. Beznosko, G. Blazey, A. Dyshkant, K. Francis, D. Kubik, V. Rykalin, M. A. Tartaglia, V. Zutshi
}

\begin{abstract}
The experimental results on the performance of the extruded scintillator and WLS fiber, and various LEDs in the magnetic fields of $1.8 \mathrm{~T}$ and $2.3 \mathrm{~T}$ respectively, are reported. The methodic used is being described
\end{abstract}

\section{INTRODUCTION}

Zuture detectors (like Digital Hadron Calorimeter [1] [2]

for a future $\mathrm{e}^{+} \mathrm{e}^{-}$linear collider) may require the use of scintillating cells with embedded photodetectors, immersed in a strong magnetic field, and the LEDs for the calibration purposes. This imposes constraints on the performance of the scintillator, fibers, LEDs and photodetectors in the magnetic field.

However, it is not always obvious how to measure various properties of these elements in the strong field's presence. In this paper we have concentrated on the methodic of measuring the scintillator response to UV LED light in the presence of magnetic field, i.e., the dependence of output's amplitude and area on the field was studied. In addition, the performance of various LEDs in the magnetic field was measured as well.

\section{EXPERIMENTAL SECTION - LEDS}

\section{A. Photodetector Description}

To measure the LED response dependence on field strength, the MRS [3] photosensor was used. The MRS photodiode is a multi-pixel solid-state device with every pixel operating in the limited Geiger multiplication mode. A resistive layer on the sensor surface achieves avalanche quenching. Due to the fact that the thickness of the active layer of this sensor is about 7 microns, MRS is shown to be non-sensitive to the magnetic field [4].

Manuscript received March 31, 2005. This work was supported in part by the U.S. Department of Education under Grant No. P116Z010035, the Department of Energy, and the State of Illinois Higher Education Cooperation Act.

M.A. Tartaglia is with Fermi National Accelerator Laboratories, Batavia, IL 60510 USA (telephone: 630-840-3890, email: tartaglia@fnal.gov).

D. Beznosko, G. Blazey, A. Dyshkant, K. Francis, D. Kubik, V. Rykalin, V. Zutshi are with Northern Illinois University, DeKalb, IL 60115 USA (telephone: 815-753-3504, email: dyshkant@fnal.gov).

\section{B. Magnet Description}

A GMW Helmholtz Dipole Magnet, model 3474, by GMW Associates [5], was used. The diameter of the poles is $250 \mathrm{~mm}$, with maximum current of 140 Amps at 76 Volts $(10.6 \mathrm{~kW})$ while water-cooled. The field strength is dependent on the distance between the poles. Due to this limitation, the highest field achieved was $2.3 \mathrm{~T}$.

\section{LED List}

The following LEDs were measured:

1. Bivar [6] LED5-UV-400-30 T1 3/4 5mm UV LED with peak emission at $400 \mathrm{~nm}$;

2. Lumex [7] SSL-DSP5093USBC Ultra Blue with peak emission at $475 \mathrm{~nm}$, and SSL-LX5093UPGC/C Ultra Pure Green with peak emission at $525 \mathrm{~nm}$;

3. Radioshack [8] 276-351 T-1-3/4 (5mm) Yellow LED with peak emission at $587 \mathrm{~mm}, 276-3205 \mathrm{~mm}$ White LED with peak emission not listed, and 276-041 $5 \mathrm{~mm}$ Red LED with peak emission at $700 \mathrm{~nm}$.

\section{LED Assembly Description}

For this test, MRS sensor was used, arranged such that the light from the LED was incident directly onto the photosensitive area via small aperture. The sensor was biased at $30.0 \mathrm{~V}$ that is well within its operating range.

Fig. 1a shows the photograph of the assembly, Fig. 1b shows its schematic and Fig. 1c is the detailed assembly photograph.

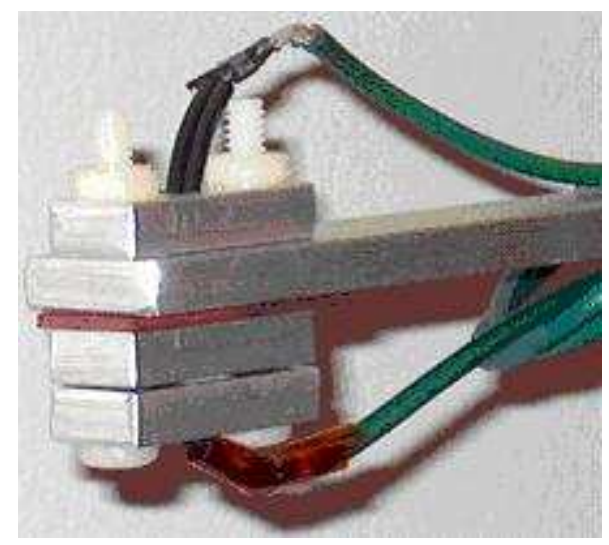

Fig. 1a. LED assembly. 


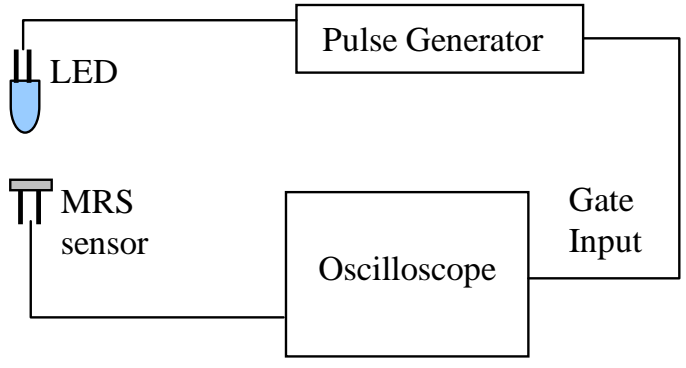

Fig. 1b. LED assembly schematic.

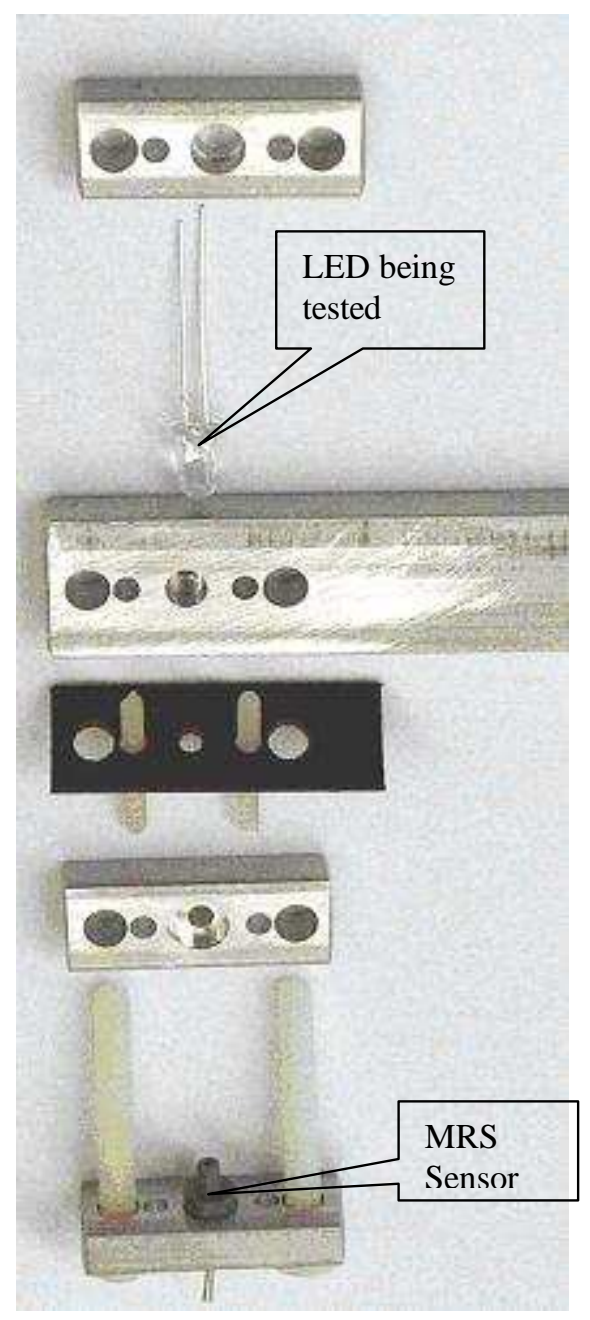

Fig. 1c. LED assembly in details.

The light pulse was produced by the easily changeable LED. The pulse from the pulse generator was $\sim 30 \mathrm{~ns}$ wide. Different amplitudes for various LEDs were needed. The output of the MRS sensors was measured and recorded by Agilent [9] Infiniium54832D MSO oscilloscope without additional preamplifier.

\section{E. LED Experimental Results}

The data for all LEDs listed in section $\mathrm{C}$ were obtained. Because of the similarity of the results, data only for Bivar UV LED will be presented for illustrative purposes. Bivar LED is of special interest since it doesn't change the spectral characteristics of its light output with change in current [10].

Here, the field is perpendicular to the LED. The amplitude and the area of the output were measured. Measurements were carried out at 0T, maximum field, 0T, maximum field again, OT, etc. The repeated measurements at 0T are conducted in order to eliminate unknown factors like the possible temperature or time changes during the experiment. In addition, the field was increased and decreased as fast as possible. Fig. 2 is the superposition of the MRS output for Bivar UV LED at OT and successive measurement at 2.3T. The full output amplitude is $\sim 124 \mathrm{mV}$ in both measurements, with area $4.7 \mathrm{nVs}$

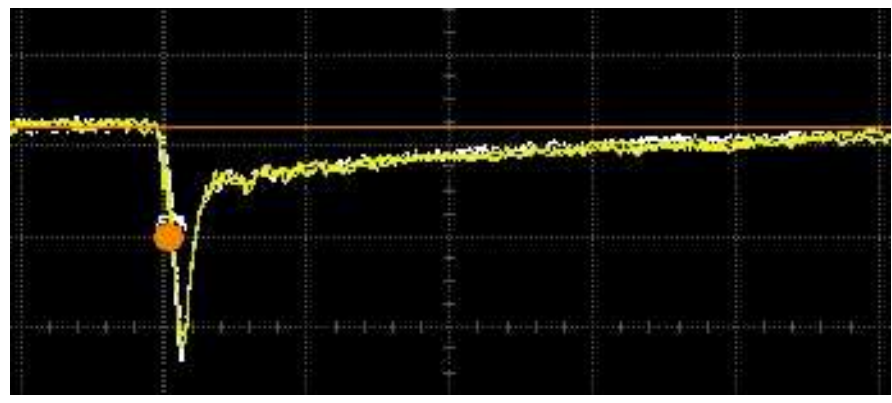

Fig. 2. Superposition of the outputs at the field strengths of 0T (white) and $2.3 \mathrm{~T}$ (yellow). The scale is $50 \mathrm{mV}$ per cell on vertical axis and $50 \mathrm{~ns}$ per cell on horizontal

From Fig. 2 there are no immediate indications of differences in the output that could be easily seen by the eye. Fig. 3 shows the values of the area of MRS output for Bivar UV LED different magnetic field strengths.

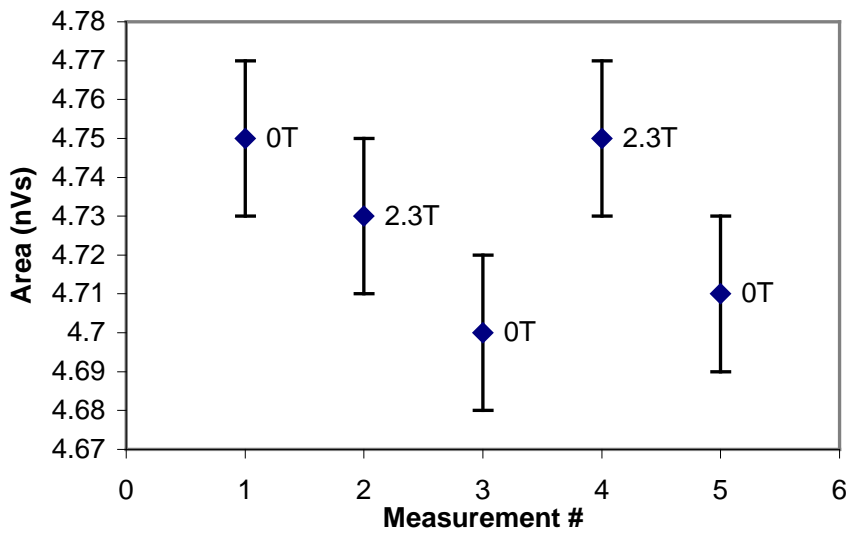

Fig. 3. Area of the MRS output for Bivar UV LED. 
The area is a measure of total charge of the output with a $50 \Omega$ load that is dependent on the total amount of incident light. Each point is an average of at least few hundred measurements. The errors are given directly by the oscilloscope. The biggest difference between points at 0T and $2.3 \mathrm{~T}$ is $\sim 1 \%$ that is within the measurement error.

Fig. 4 shows the values of the amplitude of MRS output for Bivar UV LED different magnetic field strengths. The amplitude is a measure of the peak current of the output with a $50 \Omega$ load that is dependent on the peak light output from the LED. The maximum of $\sim 1 \%$ change in output amplitude between field strength values $0 \mathrm{~T}$ and $2.3 \mathrm{~T}$ is observed.

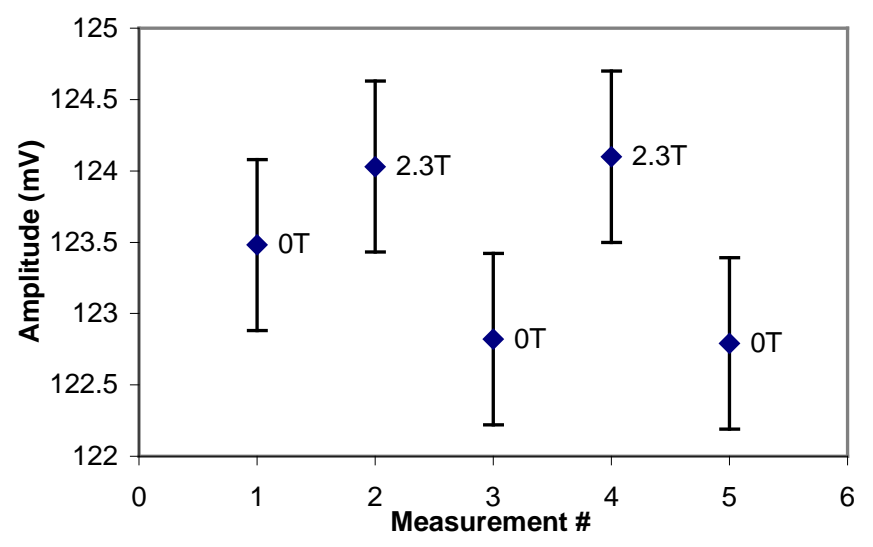

Fig. 4. Amplitude of the MRS output for Bivar UV LED.

Similar results are observed for the field being parallel to the UV LED (here, due to assembly dimensions (Fig. 1a), the maximum achievable field was $1.8 \mathrm{~T}$ ). Analogous results (Table 1) are observed for Lumex and Radioshack LEDs in the perpendicular field (2.3T) as well. Note that sometimes the maximum value of output will be at $0 \mathrm{~T}$ and sometimes at 2.3T.

TABLE 1

LED RESPONSE CHANGE IN THE MAGNETIC FIELD

\begin{tabular}{||c|c|c||}
\hline LED TESTED & $\begin{array}{c}\text { CHANGE IN } \\
\text { OUTPUT (\%) }\end{array}$ & COLOR \\
\hline SSL-DSP5093USBC & 1.4 & $\begin{array}{c}\text { Blue } \\
\text { Superbright }\end{array}$ \\
\hline SSL-LX5093UPGC/C & 0.7 & $\begin{array}{c}\text { Green } \\
\text { Suberbright }\end{array}$ \\
\hline $276-351 \mathrm{~T}-1-3 / 4$ & 1.6 & $\begin{array}{c}\text { Yellow } \\
\text { Bright }\end{array}$ \\
\hline $276-320 \mathrm{~T}-1-3 / 4$ & 1.5 & $\begin{array}{c}\text { White } \\
\text { Bright }\end{array}$ \\
\hline $276-041 \mathrm{~T}-1-3 / 4$ & 1.2 & $\begin{array}{c}\text { Red } \\
\text { Regular }\end{array}$ \\
\hline
\end{tabular}

Fig. 5a. Scintillator assembly. Red line indicates the terminal position of the WLS fiber when inserted, so that it doesn't pick up light directly from the UV LED.

With UV LED and the MRS photosensor both being insensitive of the $\mathrm{B}$ field presence, one can carry out the measurements for the scintillator in the magnetic field. Even though the thorough measurements of various scintillators was done earlier [11], we have carried out the measurements for the newly available and not yet tested in the B field extruded scintillator [12] with the Kuraray [13] Y11 1mm diameter WLS fiber embedded in the co-extruded hole.

\section{A. Scintillator Assembly Description}

For this test, $10 \mathrm{~cm} \times 2 \mathrm{~cm} \times 1 \mathrm{~cm}$ scintillator bar covered by reflective material and with WLS fiber inserted into the coextruded hole, Bivar [6] UV LED and the MRS sensor were used, arranged such that the light from the LED was incident directly onto the scintillator only. This way the WLS fiber would pick up the light only from the scintillator itself and not from the UV LED. The MRS sensor was placed in contact with the free end of the fiber, and was biased at $30.0 \mathrm{~V}$ that is well within its operating range.

Fig. 5a shows the photograph of the assembly with WLS fiber not inserted. Note that the fiber terminal position was above the LED and is indicated by the red line on the figure. Fig. 5b shows its schematic of the assembly. Fig. 5c shows the assembly between the magnet poles.

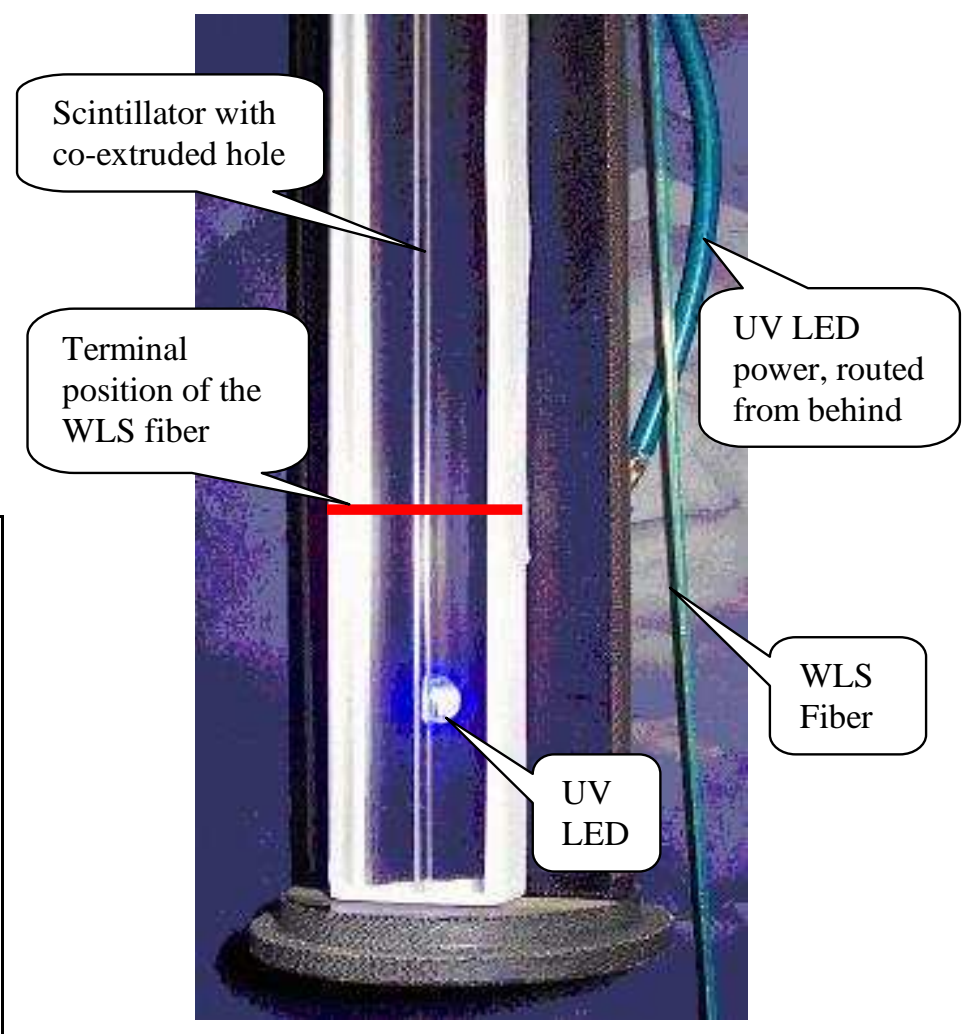




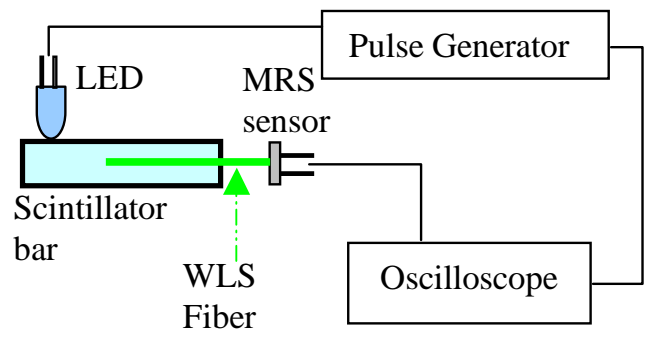

Fig. 5b. Scintillator assembly schematic.

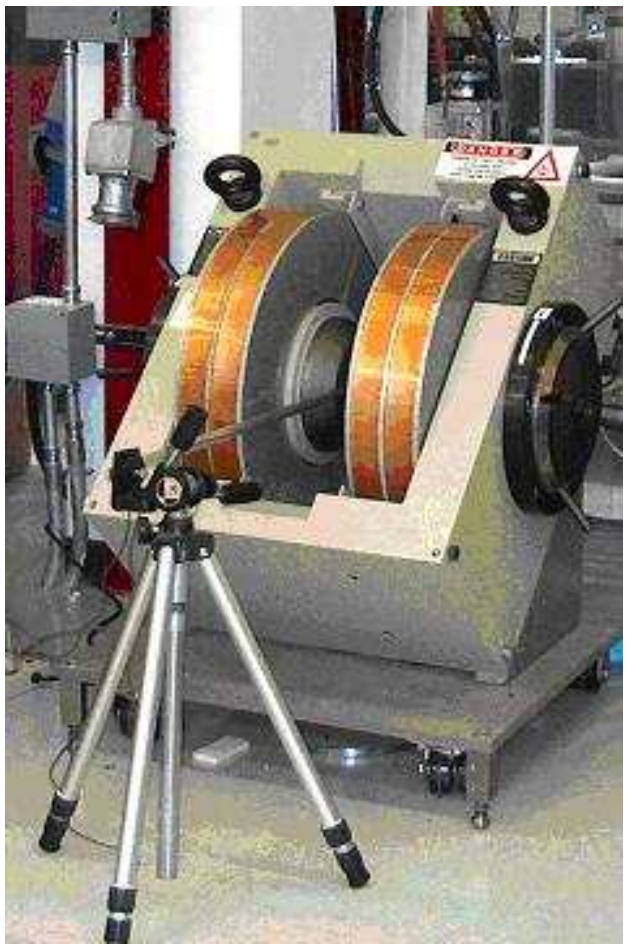

Fig. 5c. Scintillator Assembly between the magnet poles.

The pulse to the LED from the pulse generator was $~ 30 \mathrm{~ns}$ wide. The output of the MRS sensors was measured and recorded by Agilent [9] Infiniium54832D MSO oscilloscope without additional preamplifier.

\section{B. Experimental Results}

Here, the field is parallel to the LED (i.e. perpendicular to the fiber). The amplitude and the area of the output were measured. Measurements again were carried out at 0T, maximum field, 0T, maximum field again, 0T, etc. The repeated measurements at $0 \mathrm{~T}$ are conducted in order to eliminate unknown factors like the possible temperature changes during the experiment, since no thermometer was used to check the temperature. Time was given for temperature to stabilize inside the assembly ( $\sim 20$ minutes), but some temperature shift is still observed, probably due to the fact that the temperature of magnet is not constant while working. Fig. 6 is the superposition of the MRS output for extruded scintillator at $0 \mathrm{~T}$ and successive measurement at $1.8 \mathrm{~T}$. The full output amplitude here is $\sim 129 \mathrm{mV}$ in both measurements, with area $8.4 \mathrm{nVs}$

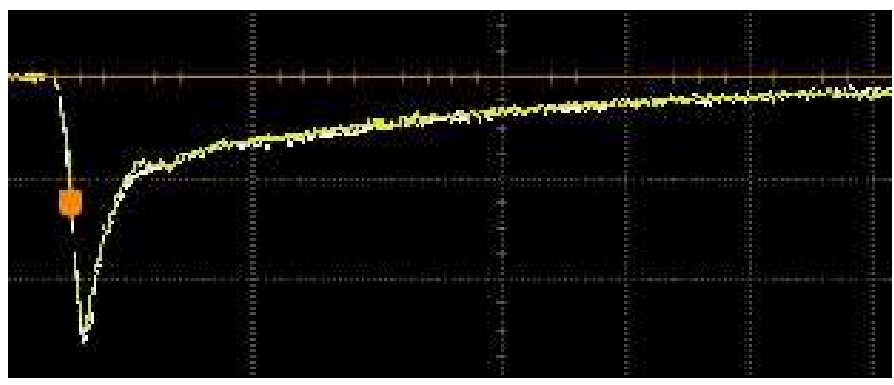

Fig. 6. Superposition of the outputs at the field strengths of 0T (white) and $1.8 \mathrm{~T}$ (yellow). The scale is $50 \mathrm{mV}$ per cell on vertical axis and 50ns per cell on horizontal. Due to technical reasons not all the cells might be visible.

From Fig. 6 there are no immediate indications of differences in the output that could be easily seen by the eye. Fig. 7 shows the values of the area of MRS output for extruded scintillator at different magnetic field strengths.

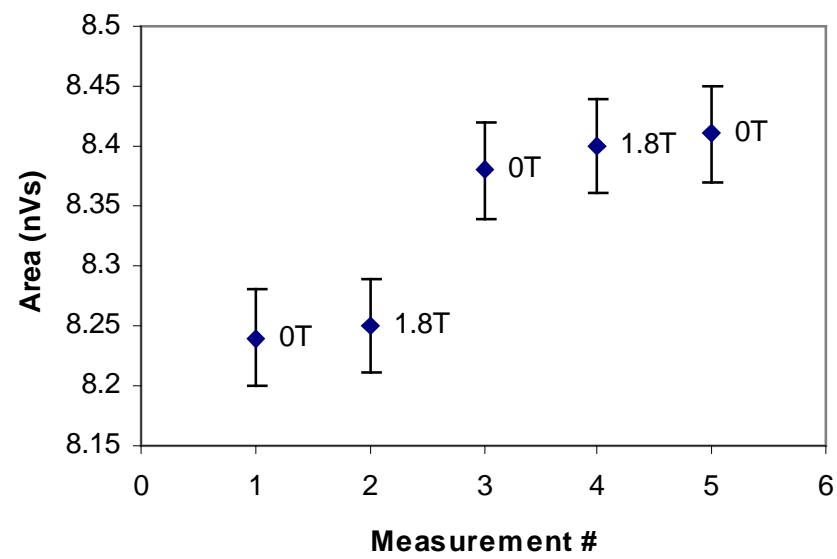

Fig. 7. Area of the MRS output for extruded scintillator.

The area is a measure of total charge of the output with a $50 \Omega$ load that is dependent on the total amount of incident light that depends on any changes in scintillator properties. Each point in every figure is an average of at least few hundred measurements at each field strength value. The errors are given directly by the oscilloscope. The biggest difference between points at $0 \mathrm{~T}$ and $1.8 \mathrm{~T}$ is $<1 \%$ counting in the fact that between first two point and the remaining three the temperature of the assembly was changed as indicated by the measurements taken 
at $0 \mathrm{~T}$, and the difference should be calculated using points from each group only.

Fig. 8 shows the values of the amplitude of MRS output for extruded scintillator at different magnetic field strengths. The amplitude is a measure of the peak current of the output with a $50 \Omega$ load that is dependent on the peak light output from the scintillator. The maximum of $<1 \%$ change in output amplitude between field strength values $0 \mathrm{~T}$ and $1.8 \mathrm{~T}$ is observed. Once again, the temperature of the assembly was changed between first two point and the remaining three measurements and difference should be calculated using points from each group

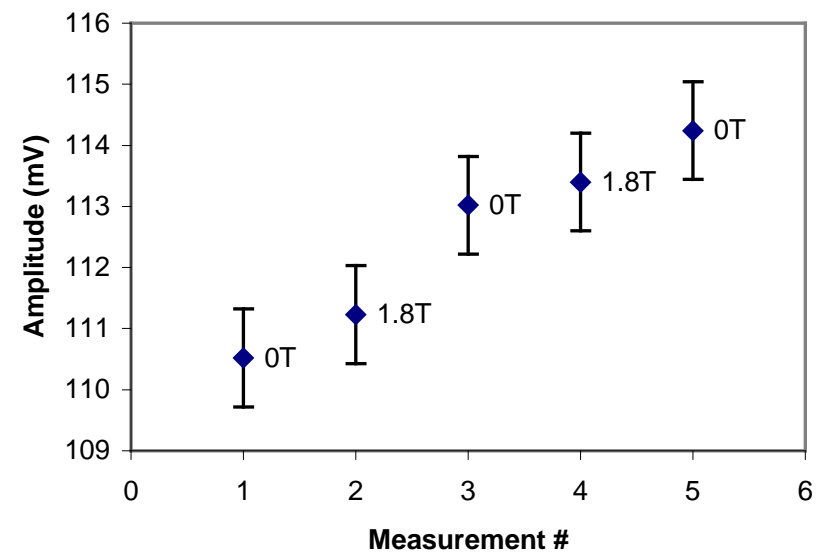

Fig.8. Amplitude of the MRS output for extruded scintillator.

\section{CONCLUSIONS}

Measurements performed using various LEDs in the magnetic field point to the insensitivity of the LED's light output on the field strength of up to $2.3 \mathrm{~T}$ within $1 \%$. This result allows using UV LED in conjunction with MRS sensors to measure the properties of the extruded scintillator in the magnetic field. The results of this measurement indicate the insensitivity of the light output levels of extruded scintillator to the magnetic fields up to $1.8 \mathrm{~T}$ within $1 \%$ when excited by the UV LED.

\section{ACKNOWLEDGEMENT}

The authors would like to thank Eugene Fisk for useful advice, Roger Nehring for his help, and Larry Gregersen for providing excellent mechanical and machining support.

\section{REFERENCES}

[1] A. Dyshkant, D. Beznosko, G. Blazey, D. Chakraborty, K. Francis, D. Kubik et al., "Towards a Scintillator-Based Digital Hadron Calorimeter for the Linear Collider Detector", IEEE vol. 51, no. 4, pp.1590-1595, Aug. 2004.

[2] A. Dyshkant, D. Beznosko, G. Blazey, D. Chakraborty, K. Frances, D. Kubik et al, "Small Scintillating Cells as the Active Elements in a Digital Hadron Calorimeter for the e+e- Linear Collider Detector", FERMILABPUB-04/015, Feb 9, 2004

[3] D. Beznosko, G. Blazey, A. Dyshkant, K. Francis, D. Kubik, A. PlaDalmau et al., "MRS Photodiode", FERMILAB-CONF-04-210-E, September 15, 04

[4] D. Beznosko, G. Blazey, A. Dyshkant, K. Francis, D. Kubik, V. Rykalin et al, "MRS Photodiode in Strong Magnetic Field", FERMILAB-TM2284, Dec. 032004

[5] GMW Associates, 955 Industrial Road, San Carlos, CA 94070

[6] Bivar Inc., 4 Thomas, Irvine, CA 92618, USA

[7] Lumex, Inc., 290 East Helen Road Palatine, IL 60067, USA.

[8] RadioShack Corporation, Riverfront Campus World Headquarters, 300 RadioShack Circle, Fort Worth, TX 76102-1964, USA.

[9] Agilent Technologies, Inc. Headquarters, 395 Page Mill Rd., Palo Alto, CA 94306, United States.

[10] P. Adamson, J. Alner, B. Anderson, T. Chase, P.J. Dervan, T. Durkin et al., "The MINOS light-injection calibration system," NuMI-PUB-SCINT, FD_DOCS-0743, NIM A 492 (2002) 325-343,Oct. 21, 2002.

[11] Dan Green, Anatoly Ronzhin, Vasken Hagopian, "Magnetic Fields and Scintillator Performance", FERMILAB-TM-1937, June 1995.

[12] D. Beznosko, A. Bross, A. Dyshkant, A. Pla-Dalmau, V. Rykalin, "FNAL-NICADD Extruded Scintillator", FERMILAB-CONF-04-216-E, September 15, 04

[13] Kuraray America Inc., 200 Park Ave, NY 10166,USA; 3-1-6, NIHONBASHI, CHUO-KU, TOKYO 103-8254, JAPAN. 